\title{
THE GENERAL PROBLEM OF ANTENNA RADIATION AND THE FUNDAMENTAL INTEGRAL EQUATION, WITH APPLICATION TO AN ANTENNA OF REVOLUTION-PART I*
}

\author{
BY \\ G. E. ALBERT \\ Naval Ordnance Plant, Indianapolis \\ AND \\ J. L. SYNGE \\ Carnegie Institute of Technology
}

Introduction. The main result of Part I is the integral equation (3.17)-a general and exact equation satisfied by the current flowing in a perfectly conducting radiating antenna, the surface of which is a surface of revolution, but otherwise unrestricted except for implicit conditions of smoothness. Part II deals with the solution of that integral equation.

No use is made of vector or scalar potentials; an approach through the electromagnetic vectors appears simpler and more direct, since a radiating field is determined by the values of the tangential component of the electric vector on the inner boundary of the infinite region. ${ }^{1}$

Section 1 gives the basic equations and boundary conditions, together with some formulae connected with a dipole. Section 2 deals with the case of radiation in a finite cavity; this simpler case points the way to the theory of antenna radiation. The basic integral equation (2.6) for cavity eigen-modes is obtained, as well as the integral equation (2.4) which has the same form as the basic equation for the exterior (antenna) case. Section 3 develops the general integral equation (3.1) or (3.2) for antenna radiation, which reduces to the comparatively simple explicit form (3.17) for an antenna of revolution. ${ }^{2}$ In Sec. 4 some actual radiating systems are discussed from the standpoint that the tangential component of the electric vector determines the field.

Section 5 is devoted to a discussion of the gap. The gap is in a sense the most important feature of an antenna because, it is the source of radiant energy. (No energy passes out through the surface of a perfect conductor.) Strangely enough, the gap has received scant attention in radiation theory. The recent work of Infeld ${ }^{3}$ shows that the question of the gap is a most delicate one; a very narrow gap leads to zero impedance unless there is a compensating thinness of the antenna at the gap. Zero impedance is avoided in the present paper by keeping the gap long compared with the thickness of the antenna at the gap as in (5.7).

In Sec. 6 the unknown is changed from the current $I(z)$ to the relative current $\phi(z)$,

*Received March 31, 1947. This paper is based on a report written by one of the authors (J.L.S.), when at the University of Toronto. This report was issued in 1942 by the National Research Council of Canada. The method now used in Part I differs considerably from that used in the report.

1The importance of the tangential components of the electromagnetic vectors appears to have been first pointed out by H. M. MacDonald, Electric waves, Cambridge, 1902, p. 16.

${ }^{2}$ For other, and we believe less exact integral equations in the antenna problem, see E. Hallén, Nova Acta Reg. Soc. Sci. Upsaliensis, 11, No. 4 (1939); P. Nicolas, L’Onde électrique, 18, 193-211 (1939); L. Brillouin, Quarterly Appl. Math. 1, 201-214 (1943).

${ }^{3}$ L. Infeld, Quarterly Appl. Math. 5, 113-132 (1947). 
viz. the ratio of the current at $z$ to the current at the center of the gap. Formulae (6.5), (6.14) give the impedance $Z$ in terms of $\phi(z)$. The function $\phi(z)$ satisfies the integral equation (6.3), not essentially different from (3.17).

The thin antenna is discussed in Sec. 7. We are able to show that the current is approximately sinusoidal outside the gap (7.10). The sinusoidal distribution of current is commonly accepted, but proofs up to the present have not been satisfactory. The present method is valid for any thin antenna of revolution; it is not assumed to be cylindrical, except at the gap.

The calculations in the case of a finite gap are too complicated to carry out at present, and for simplicity the gap is assumed to be short in Sec. 8. Whatever the practical validity of the final results may be, it must be clearly understood that for theoretical validity we must have

wave length $\gg$ length of gap $\gg$ radius of antenna.

Equation (8.10) gives in compact form the principal part of the impedance of any thin antenna with such a gap anywhere in it. If the gap is taken at the center and the shape term $X_{s}$ neglected-it is negligible for a cylindrical antenna with plane ends-we get the formula obtained by the less rational method of Labus. ${ }^{4}$

In Sec. 9 the shape term is discussed, with reference to the following thin antennae: (i) a cylinder with spheroidal ends, (ii) a spheroid, (iii) a cone. It is remarkable that the half-wave spheroid has zero reactance.

Section 10 contains a method of successive approximations for dealing with any antenna, thin or thick. The first approximations for the current and impedance agree with the principal parts obtained in Sec. 7 for the thin antenna. Higher approximations have not been calculated.

The principal features of the paper may be summarized as follows:

1) Radiation problems are reduced to the solution of integral equations involving the tangential components of the electric and magnetic vectors.

2) The gap is treated more adequately than has been done before.

3) The approximately sinusoidal current distribution for a thin antenna is established.

The usually troublesome question of end effects disappears automatically in the present method.

4) The usual first approximation for the impedance of a thin antenna is obtained with the following generalizations:

(a) The antenna is not necessarily cylindrical with plane ends.

(b) The gap is not necessarily at the center.

5) A method of successive approximations is given for dealing with thick antennae.

Advice and criticism from the following is gratefully acknowledged: Professors L. Infeld, V. G. Smith, and A. F. Stevenson, and Dr. W. Z. Chien, at the University of Toronto; and Professors A. E. Heins, and A. Weinstein, at the Carnegie Institute of Technology. Computations for the graphs were made by Mr. George Succop.

1. Notation and preliminary equations. We confine our attention to simple harmonic electromagnetic fields in vacuo; the electric and magnetic vectors are the real parts of

$$
\mathbf{E} \exp (-i k c t), \quad \mathbf{H} \exp (-i k c t),
$$

'J. Labus, Hochfrequenztechnik und Elektroakustik, 41, 17-23 (1933); G. H. Brown and R. King, Proc. I. R. E., 22, 457-480 (1934). 
where $\mathbf{E}$ and $\mathbf{H}$ are vector functions of position, in general complex, $c$ is the velocity of light, and $k$ is a real constant related to the wave length $\lambda$ by $\lambda k=2 \pi$. Except for the statement of some final results, we shall use Heaviside units, so that Maxwell's equations read

$$
\begin{gathered}
-i k \mathbf{E}=\nabla \times \mathbf{H}, \quad i k \mathbf{H}=\nabla \times \mathbf{E}, \\
\nabla \cdot \mathbf{E}=0, \quad \nabla \cdot \mathbf{H}=0 .
\end{gathered}
$$

Equations (1.3) are necessarily satisfied if (1.2) are satisfied.

As regards boundary conditions, we have on the surface of a perfect conductor, with unit normal $\mathbf{n}$,

$$
\mathbf{n} \times \mathbf{E}=\mathbf{0},
$$

i.e. $\mathbf{E}_{t}=0$, where $\mathbf{E}_{t}$ is the tangential vector component of $\mathbf{E}$. It follows from the second of (1.2) that on the surface of a perfect conductor

$$
\mathbf{H} \cdot \mathbf{n}=\mathbf{0} .
$$

In dealing with an exterior problem, conditions at infinity must be stated. Instead of the usual Sommerfeld conditions, we shall take the following as conditions for outward radiation at infinity. $A$ constant $B$ exists such that at all sufficiently great distances $\rho$ from some fixed point $O$ we have

$$
\begin{aligned}
|\mathbf{E}|<B / \boldsymbol{\rho}, & |\mathbf{H}|<B / \boldsymbol{\rho}, \\
|\mathbf{E}+\mathbf{n} \times \mathbf{H}|<B / \boldsymbol{\rho}^{2}, & |\mathbf{H}-\mathbf{n} \times \mathbf{E}|<B / \boldsymbol{\rho}^{2},
\end{aligned}
$$

where $\mathbf{n}$ is the unit vector drawn from $O$ towards the point at which the field is considered.

It is clear that these conditions are invariant under bounded displacement of the point $O$; this causes a bounded change in $\rho$ and a change in $\mathbf{n}$ of order $1 / \boldsymbol{\rho}$.

The following extended Poynting's theorem, due to H. A. Lorentz, ${ }^{5}$ is basic in our work: Let $(\mathbf{E}, \mathbf{H})$ and $\left(\mathbf{E}^{\prime}, \mathbf{H}^{\prime}\right)$ be two Maxwellian fields in a finite region $R$ bounded by $a$ surface $S$, and let neither field have any singularities in $R$ or on $S$. Then

$$
\int_{S} \mathbf{n} \cdot\left(\mathbf{E} \times \mathbf{H}^{\prime}-\mathbf{E}^{\prime} \times \mathbf{H}\right) d S=0,
$$

where $\mathbf{n}$ is the unit normal vector to $S$. This is proved very easily by Green's theorem and Maxwell's equations (1.2).

Consider now the case where the region $R$ extends to infinity, and is bounded internally by one or more surfaces $S$. Then if $\Sigma$ is any large sphere of radius $\rho$ enclosing $S$, we have

$$
\int_{S} \mathbf{n} \cdot\left(\mathbf{E} \times \mathbf{H}^{\prime}-\mathbf{E}^{\prime} \times \mathbf{H}\right) d S=\int_{\Sigma} \mathbf{n} \cdot\left(\mathbf{E} \times \mathbf{H}^{\prime}-\mathbf{E}^{\prime} \times \mathbf{H}\right) d S
$$

where $\mathbf{n}$ denotes the outward unit normal vectors to $S$ and to $\Sigma$. Suppose that both fields satisfy the conditions (1.6), (1.7) for outward radiation at infinity. Then, denoting by $T_{3}$ terms of order $1 / \rho^{3}$, we have on $\Sigma$

${ }^{5}$ Cf. Frank and Mises, Differential- und Integralgleichungen der Mechanik und Physik, Vol. 2, Braunschweig, 1927, p. 576. 


$$
\begin{aligned}
\mathbf{n} \cdot\left(\mathbf{E} \times \mathbf{H}^{\prime}-\mathbf{E}^{\prime} \times \mathbf{H}\right)= & -\mathbf{n} \cdot\left[(\mathbf{n} \times \mathbf{H}) \times \mathbf{H}^{\prime}-\left(\mathbf{n} \times \mathbf{H}^{\prime}\right) \times \mathbf{H}\right]+\mathbf{T}_{\mathbf{3}} \\
= & -\mathbf{n} \cdot\left[\mathbf{H}\left(\mathbf{n} \cdot \mathbf{H}^{\prime}\right)-\mathbf{n}\left(\mathbf{H} \cdot \mathbf{H}^{\prime}\right)\right. \\
& \left.\quad-\mathbf{H}^{\prime}(\mathbf{n} \cdot \mathbf{H})+\mathbf{n}\left(\mathbf{H}^{\prime} \cdot \mathbf{H}\right)\right]+\mathbf{T}_{\mathbf{3}} \\
= & \mathbf{T}_{\mathbf{3}} .
\end{aligned}
$$

Hence, as $\rho$ tends to infinity, the integral over $\Sigma$ tends to zero, and we have this result: Let $(\mathbf{E}, \mathbf{H})$ and $\left(\mathbf{E}^{\prime}, \mathbf{H}^{\prime}\right)$ be two Maxwellian fields in an infinite region $R$ bounded internally by one or more surfaces $S$; let neither field have any singularities in $R$ or on $S$, and let each field satisfy the conditions (1.6), (1.7) for outward radiation at infinity; then

$$
\int_{\mathbf{S}} \mathbf{n} \cdot\left(\mathbf{E} \times \mathbf{H}^{\prime}-\mathbf{E}^{\prime} \times \mathbf{H}\right) d S=0 .
$$

Suppose now that there is a dipole of vector strength $\mathbf{A}$ at the point $P^{\prime}$; the field due to it at a point $P$ is

$$
\mathbf{E}^{\prime}=\nabla(\mathbf{A} \cdot \nabla \psi)+k^{2} \psi \mathbf{A}, \quad \mathbf{H}^{\prime}=i k \mathbf{A} \times \nabla \psi, \quad \psi=e^{i k P P^{\prime}} / P P^{\prime} .
$$

This field plays an impartant part in our work. Its essential features are: (i) it satisfies Maxwell's equations, (ii) it has a pole of a certain type at $P^{\prime}$, (iii) it satisfies the conditions (1.6), (1.7) at infinity. Let $(\mathbf{E}, \mathbf{H})$ be any Maxwellian field, regular in the neighborhood of $P^{\prime}$, and let $\sigma$ be a spherical surface with center at $P^{\prime}$ and small radius $\rho$. Then

$$
\lim _{\rho \rightarrow 0} \int_{\sigma} \mathbf{n} \cdot\left(\mathbf{E} \times \mathbf{H}^{\prime}-\mathbf{E}^{\prime} \times \mathbf{H}\right) d \boldsymbol{S}=4 \pi i k \mathbf{E} \cdot \mathbf{A},
$$

where $\mathbf{E}$ on the right hand side is evaluated at $P^{\prime}$, and $\mathbf{n}$ is the unit outward normal to $\sigma$. This limit was incorrectly calculated by Sommerfeld; ${ }^{6}$ the error has been pointed out by A. F. Stevenson in a paper in course of publication, ${ }^{*}$ and the correct limit (1.13) given.

2. The integral equation for cavity radiation. Interior (cavity) and exterior (antenna) problems in electromagnetic radiation present some common features and some striking

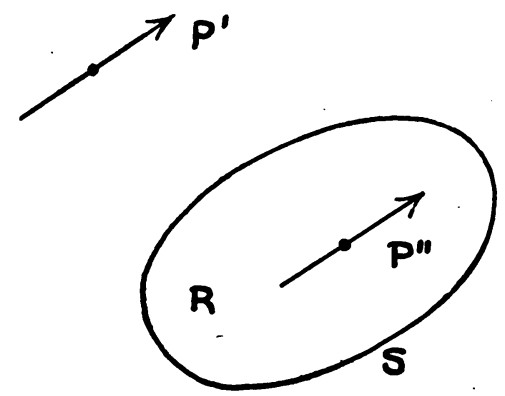

Fig. 1a. Interior Problem: Dipole at $P^{\prime}$ yields integral equation. Dipole at $P^{\prime \prime}$ yields field at $P^{\prime \prime}$.

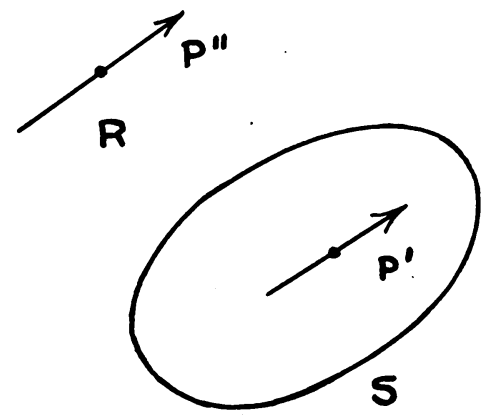

Frg. 1b. Exterior Problem: Dipole at $P^{\prime}$ yields integral equation. Dipole at $P^{\prime \prime}$ yields field at $P^{\prime \prime}$.

${ }^{6}$ P. Frank and R. v. Mises, op. cit., 578-580.

${ }^{*}$ Footnote added in proof: A. F. Stevenson, Q. Appl. Math. 5, 369-384 (1948), in particular p. 377. 
differences. Although the problem with which we are primarily concerned is the problem of antenna radiation, we shall first discuss cavity radiation.

Let $R$ be a finite region (cavity) bounded by a surface $S$ (Fig. 1a). Let (E, H) be a Maxwellian field inside $R$, satisfying (1.2); so far we do not mention boundary conditions. Let $\left(\mathbf{E}^{\prime}, \mathbf{H}^{\prime}\right)$ be the field due to a dipole situated at $P^{\prime}$, outside $R$. Then, as in (1.8),

$$
\int_{s} \mathbf{n} \cdot\left(\mathbf{E} \times \mathbf{H}^{\prime}-\mathbf{E}^{\prime} \times \mathbf{H}\right) d S=0 .
$$

Let us write

$$
\tilde{\mathbf{E}}_{t}=\mathbf{n} \times \mathbf{E}, \quad \tilde{\mathbf{H}}_{t}=\mathbf{n} \times \mathbf{H},
$$

so that $\tilde{\mathbf{E}}_{t}, \tilde{\mathbf{H}}_{t}$ are vectors tangent to $S$; they are respectively the tangential vector components $\mathbf{E}_{t}, \mathbf{H}_{t}$ of $\mathbf{E}, \mathbf{H}$, turned through a right angle about $\mathbf{n}$ in the positive sense. Then (2.1) may be written

$$
\int_{S}\left(\tilde{\mathbf{E}}_{t} \cdot \mathbf{H}^{\prime}+\tilde{\mathbf{H}}_{t} \cdot \mathbf{E}^{\prime}\right) d S=\mathbf{0}
$$

or

$$
\int_{S}\left(\tilde{\mathbf{E}}_{t} \cdot \mathbf{H}_{t}^{\prime}+\tilde{\mathbf{H}}_{t} \cdot \mathbf{E}_{t}^{\prime}\right) d S=0,
$$

where $\mathbf{E}_{t}^{\prime}, \mathbf{H}_{t}^{\prime}$ are the tangential vector components of the dipole field.

Noting that the vector strength and the position of the dipole are arbitrary (subject only to the condition that it must lie outside $R$ ), we see that (2.4) is an integral equation satisfied by the tangential components on $S$ of any Maxwellian field regular inside $S$.

Once $\left(\tilde{\mathbf{E}}_{t}, \tilde{\mathbf{H}}_{t}\right)$ are known on $S$, we can find the field $(\mathbf{E}, \mathbf{H})$ at any point $P^{\prime \prime}$ in the cavity as follows. We set up a dipole of strength $\mathbf{A}$ at $P^{\prime \prime}$; let $\left(\mathbf{E}^{\prime \prime}, \mathbf{H}^{\prime \prime}\right)$ be the field of this dipole. Then we apply (2.1) to the region bounded outside by $S$ and inside by a small sphere centered at $P^{\prime \prime}$. Then, if $\mathbf{n}$ is the unit normal to $S$, drawn into the cavity, we have by (1.13)

$$
4 \pi i k(\mathbf{E})_{P} \cdot \mathbf{A}=-\int_{S}\left(\tilde{\mathbf{E}}_{t} \cdot \mathbf{H}_{t}^{\prime \prime}+\tilde{\mathbf{H}}_{t} \cdot \mathbf{E}_{t}^{\prime \prime}\right) d S .
$$

The integral equation (2.4) may be used to study forced oscillations of a cavity, or free oscillations. Suppose, for example, that the cavity is excited by given $\mathbf{E}_{\boldsymbol{t}}$ on its surface; then (2.4) becomes an integral equation to determine $\mathbf{H}_{t}$, and, if it can be solved, the field in the cavity is given by (2.5). In the case of free oscillations in a cavity with perfectly conducting walls, we put $\mathbf{E}_{t}=0$; then (2.4) reads

$$
\int_{S} \tilde{\mathbf{H}}_{t} \cdot \mathbf{E}_{t}^{\prime} d S=0 .
$$

It is well known that free oscillations in a cavity are possible only for definite values (eigenvalues) of $k ;(2.6)$ is an integral equation of an unusual type for the determination of such eigenvalues. But we shall not discuss the question of eigenvalues here. ${ }^{7}$

${ }^{7}$ Cf. H. Weyl, Journal für Mathematik, 143, 177-202 (1914); Rendiconti del Circolo Matematico id Palermo, 39, 1-50 (1915). 
The reader should remember that, in the above work,

$\left(\mathbf{E}^{\prime}, \mathbf{H}^{\prime}\right)$ = field due to dipole outside the cavity;

$\left(\mathbf{E}^{\prime \prime}, \mathbf{H}^{\prime \prime}\right)=$ field due to dipole placed in the cavity.

3. The integral equation for antenna radiation. Let $R$ be an infinite region bounded internally by a surface $S$. We are interested in simply harmonic Maxwellian fields, satisfying the conditions (1.6), (1.7) of outward radiation at infinity and certain conditions over the surface $S$ (Fig. 1b). In the physical problem, $S$ is a surface enclosing the antenna or coincident with it.

Let $(\mathbf{E}, \mathbf{H})$ be the field under discussion. Let $\left(\mathbf{E}^{\prime}, \mathbf{H}^{\prime}\right)$ be the field of a dipole of vector strength $\mathbf{A}$ situated at any point $P^{\prime}$ inside $S$, i.e. not in the region $R$. We may apply the result (1.8) to the surface composed in part of $S$ and in part of a large sphere. As we have already seen, the contribution from the large sphere tends to zero as its radius tends to infinity. Hence, in the notation of (2.4) we have

$$
\int_{S}\left(\tilde{\mathbf{E}}_{t} \cdot \mathbf{H}_{t}^{\prime}+\tilde{\mathbf{H}}_{t} \cdot \mathbf{E}_{t}^{\prime}\right) d S=0,
$$

where $t$ signifies "tangential vector component" and $\tilde{\mathbf{E}}_{t}, \tilde{\mathbf{H}}_{t}$ denote that $\mathbf{E}_{t}, \mathbf{H}_{t}$ are turned through a right angle in the positive sense about the unit normal $\mathbf{n}$ to $S$. We shall in future regard $\mathbf{n}$ as drawn out from $S$, i.e. into $R$. It is clear that $\tilde{\mathbf{E}}_{t} \cdot \mathbf{H}_{t}^{\prime}=-\mathbf{E}_{t} \cdot \tilde{\mathbf{H}}_{t}^{\prime}$, and so (3.1) may be written

$$
\int_{S} \tilde{\mathbf{H}}_{t} \cdot \mathbf{E}_{t}^{\prime} d S=\int_{S} \mathbf{E}_{t} \cdot \tilde{\mathbf{H}}_{t}^{\prime} d S .
$$

Equation (3.1) or (3.2) is the fundamental integral equation of antenna theory. If this equation is solved, and $\mathbf{E}_{t}, \mathbf{H}_{t}$ found, the field at any point in $R$ is obtained by taking a dipole of vector strength $\mathbf{A}$ at any point $P^{\prime \prime}$ inside $R$, i.e. outside $S$. Then, as in (2.5), we have

$$
4 \pi i k(\mathbf{E})_{P} \cdots \cdot \mathbf{A}=-\int_{S}\left(\tilde{\mathbf{E}}_{t} \cdot \mathbf{H}_{t_{\bullet}^{\prime \prime}}+\tilde{\mathbf{H}}_{t} \cdot \mathbf{E}_{t}^{\prime \prime}\right) d S,
$$

when $\left(\mathbf{E}^{\prime \prime}, \mathbf{H}^{\prime \prime}\right)$ is the field due to the dipole placed at $P^{\prime \prime}$; the normal on $S$ points outward.

Let us now apply our equation (3.2) to the case of an antenna having symmetry of revolution about the $z$-axis (Fig. 2).

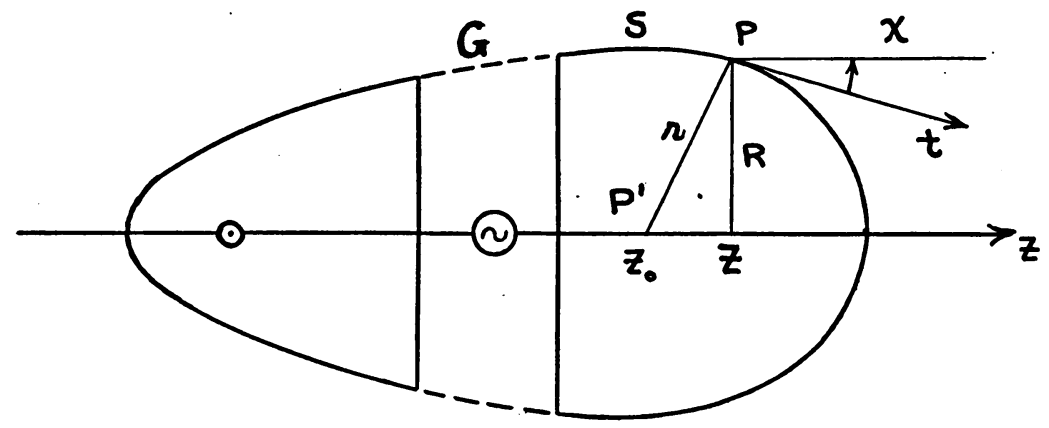

Fig. 2. Antenna of revolution with gap. 
The antenna is broken by a gap containing a generator. Let the surface of the antenna be extended by a mathematical surface $G$ covering the gap, and let $S$ be a closed surface consisting in part of the surface of the antenna and in part of the surface $G$ drawn over the gap. We shall suppose the excitation of the antenna to be symmetric in the sense that, on $G, \mathbf{E}_{t}$ lies in the meridian plane. It is natural, then, to consider only fields of radiation having this type of symmetry, i.e. $\mathbf{E}$ at any point $P$ lies in the meridian plane through $P$ and the $z$-axis, and $\mathbf{H}$ in consequence is perpendicular to the meridian plane. On $S, \tilde{\mathbf{H}}_{t}$ lies in the meridian plane.

Now take a dipole at any point $P^{\prime}\left(z_{0}\right)$ on the axis of the antenna inside $S$, the axis of the dipole lying along the axis of the antenna, and its strength being unity. We shall apply the fundamental equation (3.2).

Introducing cylindrical coordinates $(z, R, \omega)$, we have, by (1.12),

$$
\psi=e^{i k r} / r, \quad r^{2}=R^{2}+\left(z-z_{0}\right)^{2} .
$$

The surviving components of the field of the dipole are

$$
E_{z}^{\prime}=\frac{\partial^{2} \psi}{\partial z^{2}}+k^{2} \psi, \quad E_{R}^{\prime}=\frac{\partial^{2} \psi}{\partial R \partial z}, \quad H_{\omega}^{\prime}=i k \frac{\partial \psi}{\partial R},
$$

where the differentiations of $\psi$ are carried out with $R, z, z_{0}$ treated as independent variables. However, on the surface of the antenna, $R$ is a function of $z$, and so we may write $\psi=\psi\left(z, z_{0}\right)$. Let us temporarily employ $d$ as a symbol of partial differentiation when $\psi$ and its derivatives are regarded as functions of $z$ and $z_{0}$. Then, noting that

$$
\begin{aligned}
& \frac{d}{d z_{0}} \frac{\partial \psi}{\partial R}=\frac{\partial}{\partial z_{0}} \frac{\partial \psi}{\partial R}=-\frac{\partial}{\partial z} \frac{\partial \psi}{\partial R}, \\
& \frac{d}{d z_{0}} \frac{\partial \psi}{\partial z}=\frac{\partial}{\partial z_{0}} \frac{\partial \psi}{\partial z}=-\frac{\partial}{\partial z} \frac{\partial \psi}{\partial z},
\end{aligned}
$$

we have

$$
\begin{aligned}
\frac{d \psi}{d z} & =\frac{\partial \psi}{\partial R} \frac{d R}{d z}+\frac{\partial \psi}{\partial z}, \\
\frac{d^{2} \psi}{d z_{0} d z} & =-\frac{\partial^{2} \psi}{\partial R \partial z} \frac{d R}{d z}-\frac{\partial^{2} \psi}{\partial z^{2}} .
\end{aligned}
$$

The tangential component $\mathbf{E}_{t}^{\prime}$ may be written $\mathbf{E}_{t}^{\prime}=E^{\prime} \mathbf{t}$, where $\mathrm{t}$ is a unit tangent vector to the meridian curve, as shown in Fig. 2; $\mathrm{t}$ makes an angle $\chi$ with the $z$-axis, so that

$$
\sin \chi=-\frac{d R / d z}{\left[1+(d R / d z)^{2}\right]^{1 / 2}}, \quad \cos \chi=\frac{1}{\left[1+(d R / d z)^{2}\right]^{1 / 2}}
$$

Then, by (3.5) and (3.6),

$$
\begin{aligned}
E^{\prime} & =E_{z}^{\prime} \cos \chi-E_{R}^{\prime} \sin \chi \\
& =\left[1+(d R / d z)^{2}\right]^{-1 / 2}\left[\frac{\partial^{2} \psi}{\partial z^{2}}+k^{2} \psi+\frac{\partial^{2} \psi}{\partial R \partial z} \frac{d R}{d z}\right] \\
& =\left[1+(d R / d z)^{2}\right]^{-1 / 2}\left[k^{2} \psi-\frac{d^{2} \psi}{d z d z_{0}}\right] .
\end{aligned}
$$


If we write $\tilde{\mathbf{H}}_{t}=\tilde{H} t$, we have

$$
\tilde{\mathbf{H}}_{t} \cdot \mathbf{E}_{t}^{\prime}=E^{\prime} \tilde{H}=\left[1+(d R / d z)^{2}\right]^{-1 / 2}\left[k^{2} \psi-\frac{d^{2} \psi}{d z d z_{0}}\right] \tilde{H} .
$$

Also, we may write $\mathbf{E}_{\iota}=E \mathrm{t}, \tilde{\mathbf{H}}_{t}^{\prime}=\tilde{H}^{\prime} \mathrm{t}$, where, by (3.5),

$$
\tilde{H}^{\prime}=H_{\omega}^{\prime}=i k \frac{\partial \psi}{\partial R}
$$

Now $\psi$ is a function. of $r$, a single variable, and $d \psi / d r$ will denote the derivative. We have

$$
\frac{\partial \psi}{\partial R}=\frac{d \psi}{d r} \frac{\partial r}{\partial R}=\frac{R}{r} \frac{d \psi}{d r}
$$

and so

$$
\mathbf{E}_{t} \cdot \tilde{\mathbf{H}}_{t}^{\prime}=E \tilde{H}^{\prime}=i k E \frac{R}{r} \frac{d \psi}{d r} .
$$

Thus our basic equation (3.2) reads

$$
\int_{S}\left[k^{2} \psi-\frac{d^{2} \psi}{d z d z_{0}}\right]\left[1+(d R / d z)^{2}\right]^{-1 / 2} \tilde{H} d S=i k \int_{S} \frac{R}{r} \frac{d \psi}{d r} E d S .
$$

Let us denote by $I(z)$ the current flowing on the surface of the antenna across the section $z=$ constant. Then

$$
I(z)=2 \pi c R \tilde{H}
$$

We shall regard this as the definition of $I(z)$ on $G$. Also

$$
d S=2 \pi R\left[1+(d R / d z)^{2}\right]^{1 / 2} d z .
$$

Hence (3.13) may be written

$$
\int\left(k^{2} \psi-\frac{d^{2} \psi}{d z d z_{0}}\right) I(z) d z=2 \pi i k c \int R^{2}\left[1+(d R / d z)^{2}\right]^{1 / 2} \frac{1}{r} \frac{d \psi}{d r} E(z) d z .
$$

This equation (3.16) is the basic integral equation for the determination of the current in an antenna of revolution. We shall therefore restate it in complete form with slightly revised notation: Consider a perfectly conducting antenna having the z-axis for axis of symmetry, and excited symmetrically. Let the antenna extend from $z=l_{1}$ to $z=l_{2}$. Let $R=R(z)$ be the equation in cylindrical coordinates of a surface $S$ which consists of the surface of the antenna and the gap $G$. Let $E(z)$ be the tangential component of the electric vector on the gap, and $E(z)=0$ outside the gap. Let $I(z)$ be the current across the section $z=$ const. as given by (3.14). Then $I(z)$ satisfies the integral equation

$$
\int_{l_{1}}^{l_{2}} K\left(z, z_{0}\right) I(z) d z=i M\left(z_{0}\right), \quad l_{1}<z_{0}<l_{2} \text {. }
$$

where

$$
\begin{aligned}
K\left(z, z_{0}\right) & =-\frac{\partial^{2} \psi}{\partial z \partial z_{0}}+k^{2} \psi \\
\psi & =\psi\left(z, z_{0}\right)=e^{i k r} / r, \quad r^{2}=[R(z)]^{2}+\left(z-z_{0}\right)^{2},
\end{aligned}
$$




$$
\begin{aligned}
M\left(z_{0}\right) & =2 \pi k c \int_{l_{1}}^{l_{2}} E R^{2}\left(1+{R^{\prime 2}}^{1 / 2} \frac{1}{r} \frac{d \psi}{d r} d z,\right. \\
R^{\prime} & =d R / d z .
\end{aligned}
$$

Since confusion with regard to partial differentiation has now been removed, we have restored the usual notation, $\psi$ being regarded as a function of $z$ and $z_{0}$. It should be noted that on account of the occurrence of $R(z)$ in $r$, the kernel $K\left(z, z_{0}\right)$ is not symmetric. Since $E=0$ outside the gap, the limits of integration in the expression for $M\left(z_{0}\right)$ may be replaced by those corresponding to the ends of the gap.

4. Some radiation problems. In setting up simplified mathematical models for the treatment of radiation problems, it is essential that we carry into the model sufficient conditions to yield a definite mathematical problem, without putting in so many conditions as to make the problem insoluble. The following discussion is based on the fact that a field of outward radiation is determined by the assignment of $\mathbf{E}_{\imath}$ over a surface $S$ which forms the inner boundary of the infinite region.

The mast antenna. The common problem of macrowave radiation involves a vertical mast above a horizontal earth (Fig. 3). Let us make, the usual assumption of infinite conductivity. We think, then, of an infinitely conducting cylinder $A B$ above an infinitely conducting plane. The apparatus is fed by a generator $D$, the terminals of which

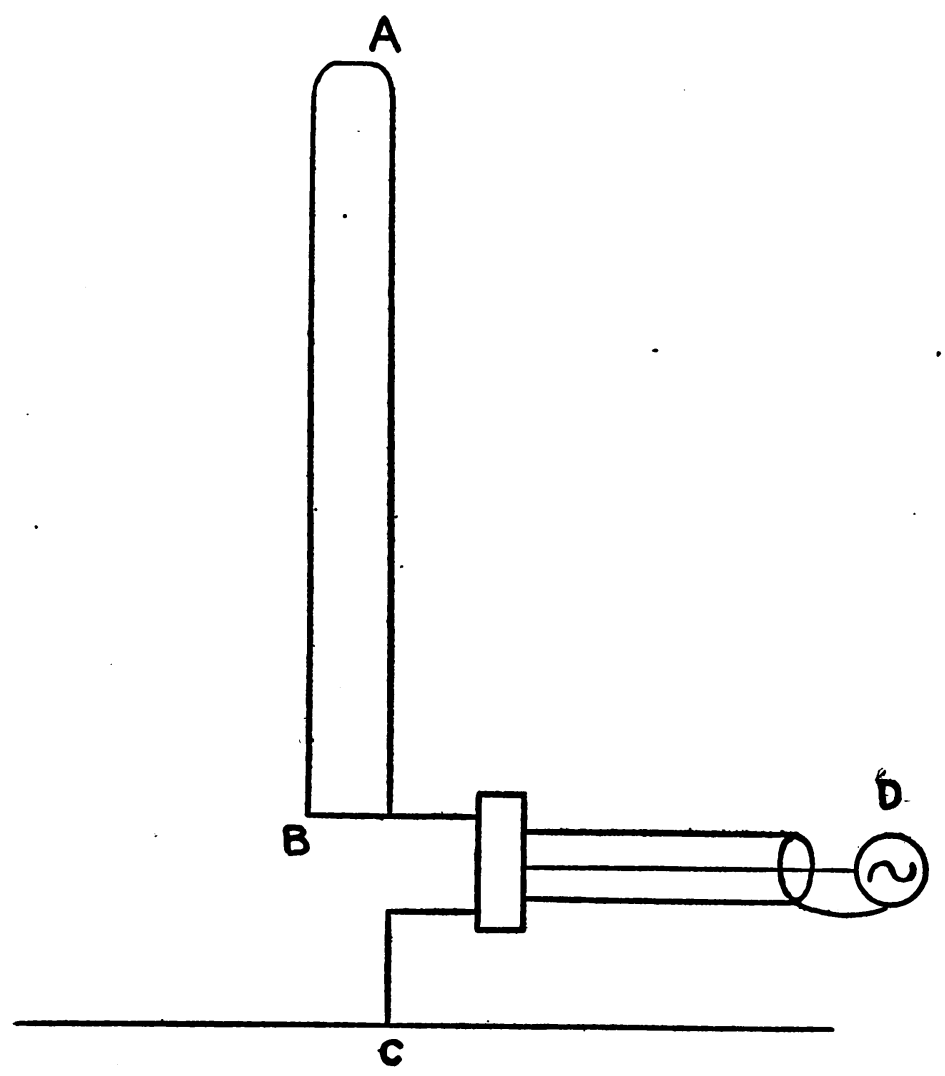

Fra. 3. The mast antenna 
are connected to $A B$ and to the plane at $C$. We have then the problem of determining a Maxwellian field satisfying the following conditions:

(i) outward radiation at infinity,

(ii) $\mathbf{E}_{t}=0$ over $A B$, over the plane earth, and over the connecting leads.

Where does the energy come from, which reaches infinity? It cannot come from the perfectly conducting surfaces, since $\mathbf{E}_{t}=0$ implies that the Poynting vector is tangent to a perfectly conducting surface. Hence the only possible source of the energy is a surface drawn around the generator $D$. (If that surface were perfectly conducting, the generator would be short-circuited, and there would be no radiation at all.) The rest of the apparatus serves only as a guide to the radiant energy.

It would appear therefore that we can obtain a determinate problem in the case of Fig. 3 only by assigning $\mathbf{E}_{t}$ over a surface which bounds the generator. If this were done, we would have $\mathbf{E}_{t}$ all over a surface consisting partly of the aforesaid surface and partly of the surfaces of the conductors, and so we would have a determinate problem.

But in the actual treatments of such problems, no reference is made to the geometry of a surface bounding the generator, nor even (usually) to the geometry of the leads. A discussion along the lines suggested above would be, apparently, unnecessarily complicated from a practical viewpoint. It is usual to simplify the problem by substituting a model as shown in Fig. 4 for the actual system. Now the generator $D$ is reduced to

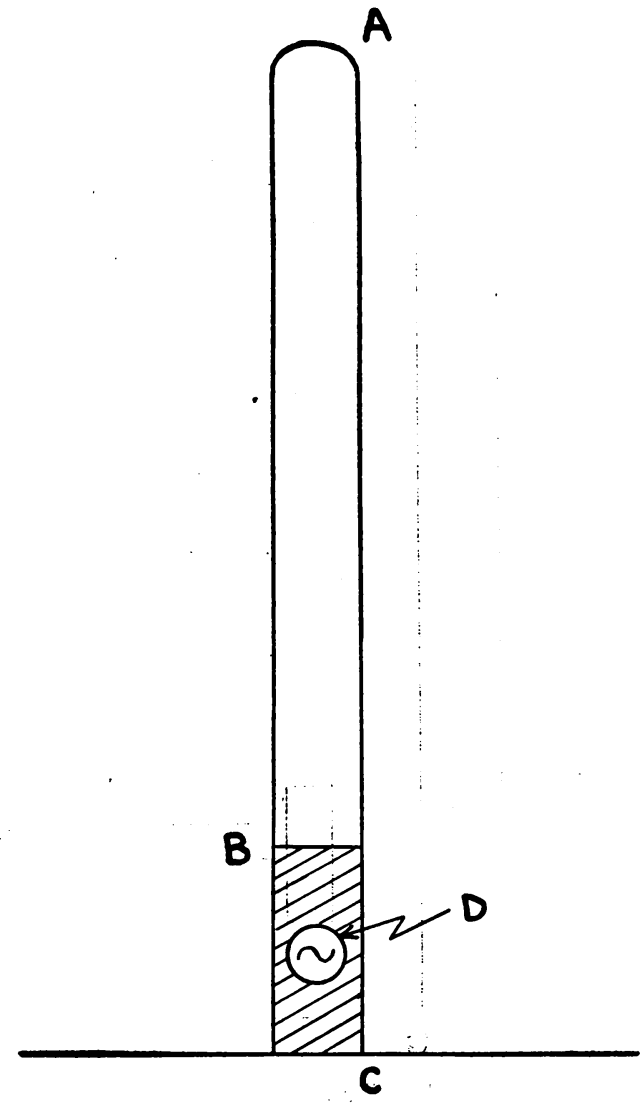

Fig. 4. The mast antenna with gap. 
small proportions and placed between the antenna and the ground. To provide a surface for boundary conditions, the geometrical surface of the antenna is continued down past the generator to the ground.

It is not, of course, suggested that the above arrangement is ever employed in practice. But it is the arrangement employed in mathematical discussions, whether the authors admit it or not. The hope is that the behavior of the model shown in Fig. 4 (deduced mathematically) will agree with the behavior of the actual apparatus of Fig. 3. On this point we have no assurance from theory.

Figure 4 introduces the gap; by this we mean the surface $B C$, bounding the generator. Many writers have proceeded as if antenna problems could be discussed without reference to a gap. To do so, however, is to leave out the essential part of a radiating system. The gap is the only source of radiant energy.

Now if we knew $\mathbf{E}_{t}$ over the gap, we would have a determinate problem in Fig. 4. But we do not know $\mathbf{E}_{t}$ over the gap. This may be expressed by saying that we do not know the (electrical) structure of the gap. But if the gap is short, its structure does not seem to be important. It is then possible to disregard the structure, and express the radiation properties of the antenna in terms of only one characteristic of the gap, namely, the voltage or potential difference. This is the integral of $\mathbf{E}_{t}$ taken along $B C$, with suitable sign.

The apparatus used by Brown and Woodward ${ }^{8}$ for the experimental measurement of impedance is similar to that shown in Fig. 4, except that their gap is shorter and the mast is fed by a coaxial line which comes in from below through the ground.

The cigar antenna. It is customary and legitimate to eliminate the earthplane in Fig: 4 by a method of symmetry. This leads to the cigar antenna (Fig. 5). This self-

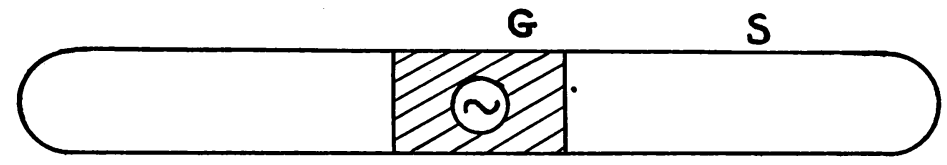

Fig. 5. The cigar antenna.

contained radiator is a mathematical fiction, but it could be realized in practice. We think of a hollow copper cigar, cut in two to leave a gap. Inside there is a generator, with terminals connected to the two ends of the cigar. (The two generators obtained by reflection of Fig. 4 in the earth are replaced by one generator in Fig. 5; but this is a triviality, because we are not interested in the interior of the cigar. What interests us is $\mathbf{E}_{t}$ over the gap $G$.)

Although the source of the cigar antenna is to be found in the application of the reflection process to the mast antenna, it suggests a generalization in which symmetry is abandoned (Fig. 6). Figure 7 shows a coaxial line terminated in a spherical knob. The model for this (Fig. 8) is the limiting case of an asymmetric cigar, one end being reduced to a small circular patch on a conducting sphere, surrounded by a ring gap.

The later detailed applications in this paper deal with a cigar antenna, in general asymmetric.

The microwave antenna terminating a coaxial line. Let us now consider a microwave

${ }^{8}$ G. H. Brown and O. M. Woodward, Proc. I. R. E., 33, 257-262 (1945). 


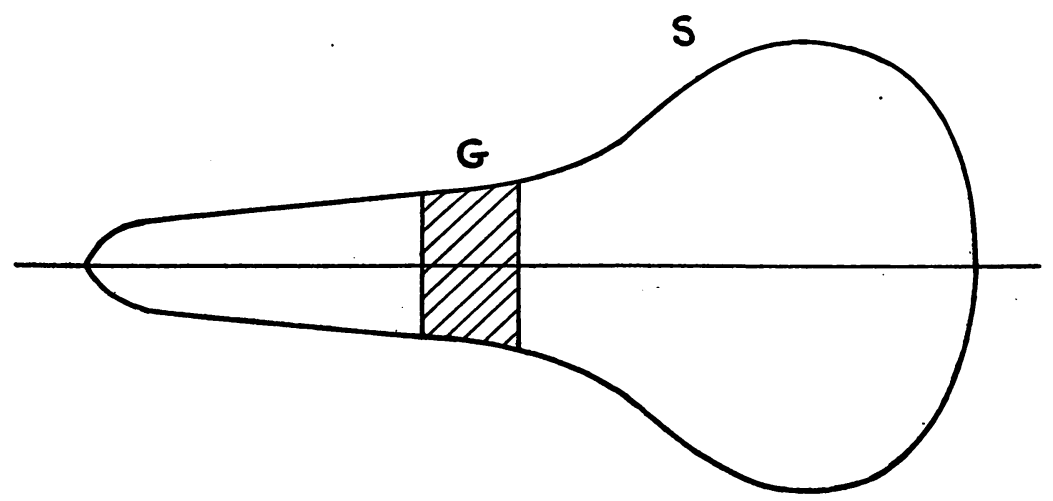

Fig. 6. The cigar antenna (unsymmetric).

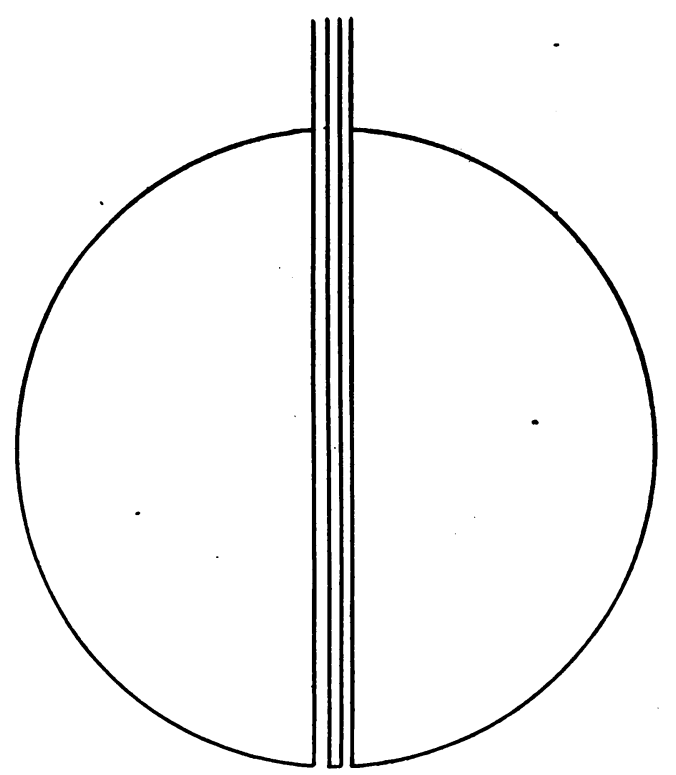

Fra. 7. Coaxial line terminated by a spherical knob.

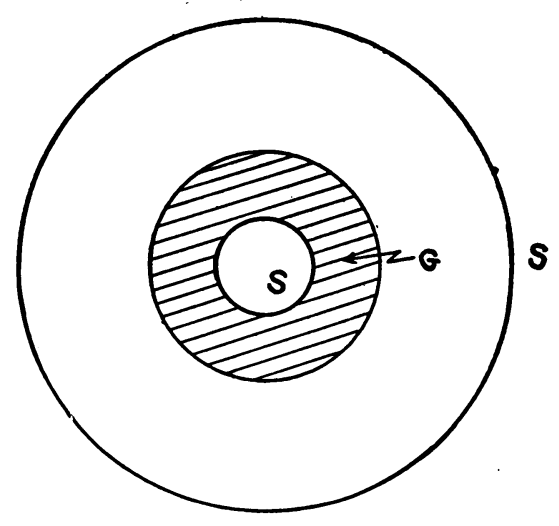

Fig. 8. Spherical antenna with ring gap.

apparatus. Figure 9 shows a coaxial line, terminated in an antenna. We may introduce the idealization that the line extends to infinity.'

Let us see whether this apparatus gives a definite problem. For surface $S$ we take the surface of the antenna and the core of the coaxial, together with the inner and outer surfaces of the sheath of the coaxial. Over this surface we have $\mathbf{E}_{t}=0$. Conditions at infinity, however, are not obvious. We cannot say that there is outward radiation in all directions-there is nowhere for this radiation to come from. We expect that there will be outward radiation everywhere except in the neighbourhood-of the coaxial line, and that inside the line there will be inward radiation (together with some outward radiation if there is incomplete tuning).

Thus, while Fig. 9 presents difficulties as far as the definition of a determinate mathematical problem is concerned, these difficulties are not those encountered previously. There is no troublesome gap-unless we like to say that the gap is at infinity. 
Obviously some simplification is necessary for mathematical attack. The usual simplification is drastic. We retain only the horizontal arms of the antenna, wiping out all the rest of the apparatus. We join the arms (dotted indication in Fig. 9) and regard this cylindrical boundary as a gap. In fact, we return to the cigar antenna! It is indeed remarkable that impedance calculations obtained from such a simplified model should have the practical value that they do seem to possess.

The idealization of the microwave antenna of Fig. 9 to the cigar antenna of Fig. 5 involves us in the old difficulties concerning the gap. This seems particularly objection-

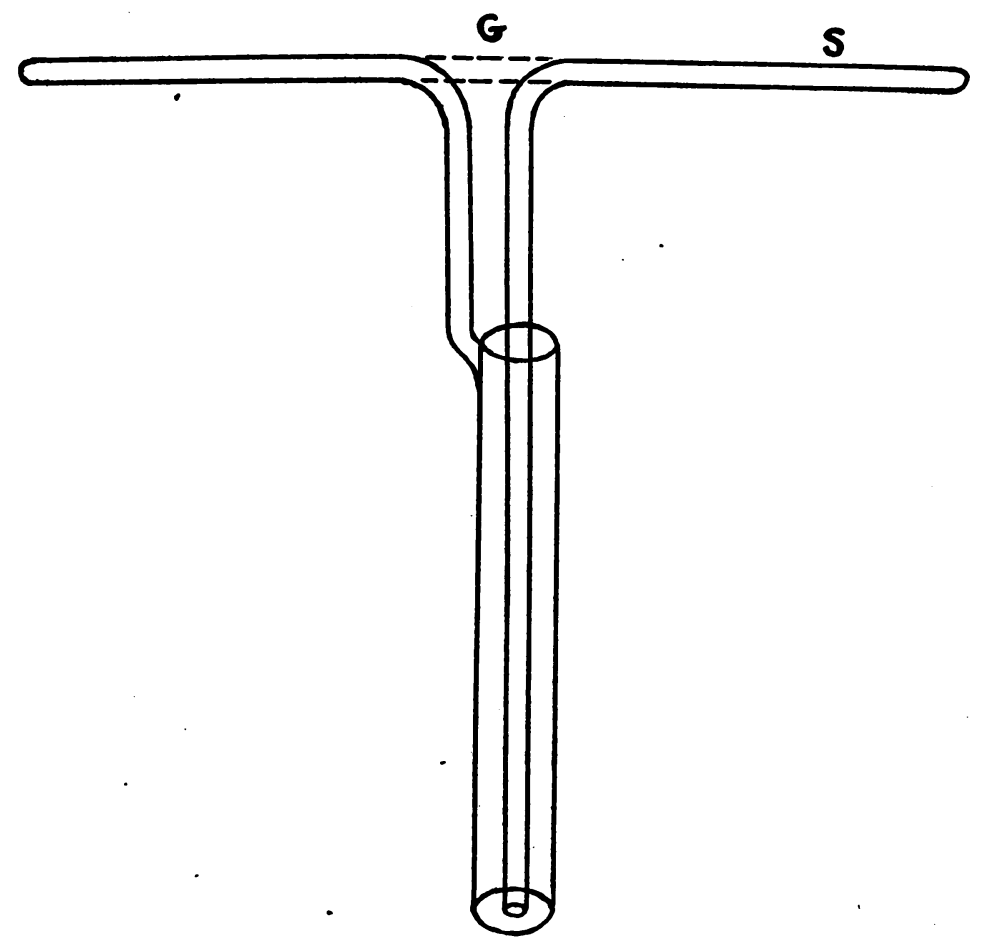

Fig. 9. Two-arm antenna terminating coaxial line.

able because in actual antennae the gap is not very small compared to the length of the antenna. Is there not some way to discuss the problem without introducing a gap artificially? It appears difficult in the case of the arrangement of Fig. 9.

With a different form of apparatus, however, the situation is more hopeful. Figure 10 shows a coaxial line, with the core projecting as an antenna, while the sheath spreads out into a conducting plane. The surface $S$ consists of the surfaces of the core and antenna, with the inner surface of the sheath and the surface of the conducting plane. Over $S$ we have $\mathbf{E}_{t}=0$. On the infinite hemisphere above the antenna we assign outward radiation. At infinity down the line we assign a direct wave (amplitude $C_{1}$ ) and a reflected wave (amplitude $C_{2}$ ). The problem of determining the ratio $C_{1} / C_{2}$ is then a definite one. We approach the important problem of reflection without using the artificial concept of impedance.

To bring the preceding problem more into line with what was said earlier regarding determination, we may change the conditions down the line. Let us say that $\mathbf{E}_{t}$ is assigned 
on a cross-section a long way down. Now we have a definite problem, since $\mathbf{E}_{t}$ is given over the bounding surface, with a condition of outward radiation in the hemisphere. If we find $\mathbf{H}_{t}$ on the section of the line, we can find the ratio of the reflected to the incident wave.

The case of Fig. 10 should therefore be treated with considerable respect, because it represents an actual situation in which the radiation problem is definite; it is unnecessary to simplify the apparatus drastically for mathematical purposes. ${ }^{9}$

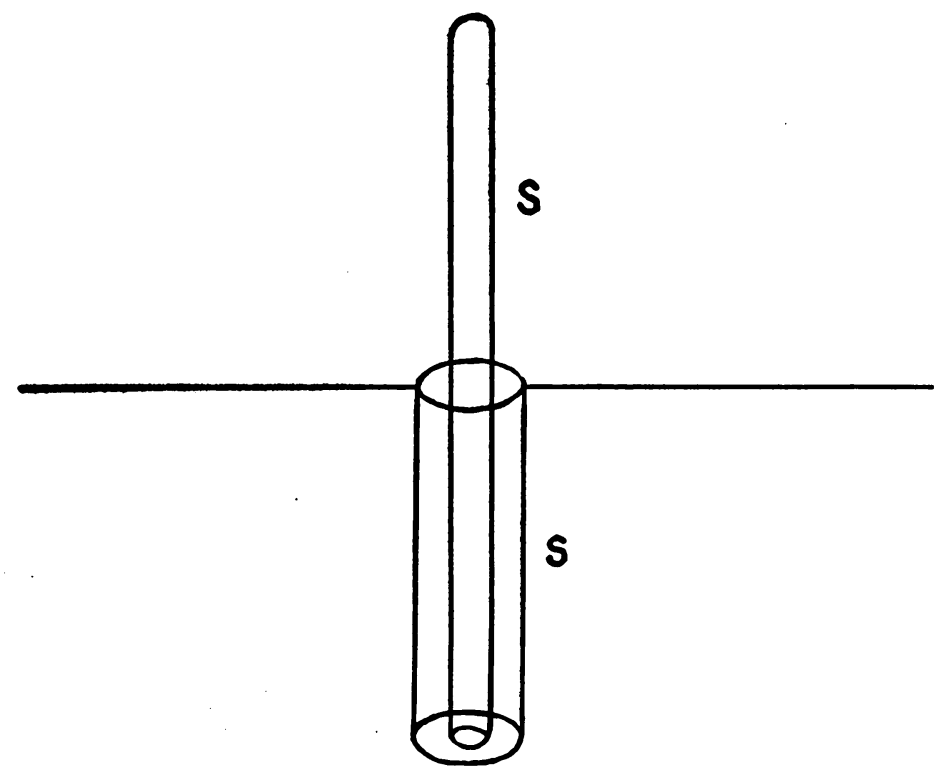

FIg. 10. Coaxial line with antenna projecting beyond conducting plate.

Before leaving the case of Fig. 10 we may mention how this apparatus may be considered as of the gap type. If we consider only phenomena above the plane of the flat conducting surface, we have a situation very like that of the conducting mast; the only difference is that the gap is now around the base of the mast instead of being below it. Once more we may appeal to the method of symmetry and discuss the problem as a cigar problem (Fig. 11). Now the surface $S$ consists of a complete cigar with a disc attached at its center. We must assume $\mathbf{E}_{t}$ given over this disc, which is in fact the gap in a new form. We are not to expect that the field has continuous derivatives if we pass through the disc. We may call Fig. 5 a cigar-with-a-band and Fig. 11 a cigar-with-a-frill.

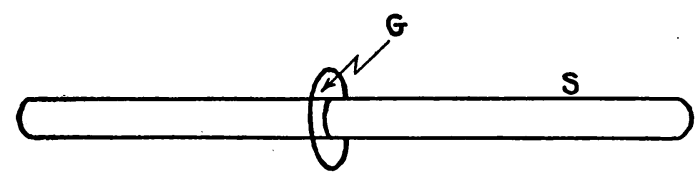

FIg. 11. Cigar with a frill.

${ }^{9}$ Experimental results for this case have been given by Brown and Woodward, loc. cit. 
The antenna in the wave-guide. Figure 12 shows an antenna in a wave-guide. This problem lends itself to the same satisfactory mathematical formulation as for Fig. 10. There are only two differences. Instead of a hemisphere at infinity above, we have the opening of the wave guide at infinity on the right, and there we impose a condition of outward radiation. (That is in the case of transmission; for reception, we must allow for a direct and reflected wave, with only a wave down the coaxial line.) Secondly, the five faces of the guide (if it is of rectangular section) increase the complexity of the geometry.

Should we wish to introduce the gap concept, we may use the method of images

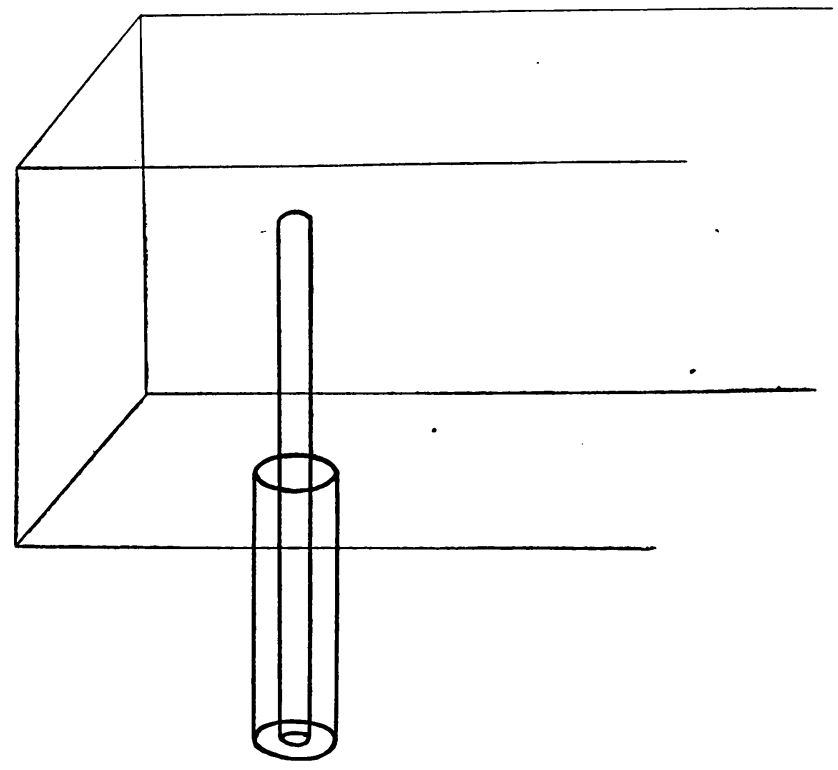

FIg. 12. Antenna in a wave-guide.

when the guide is rectangular. This means that we forget all about the walls of the guide and the coaxial line, and consider a doubly infinite pattern of cigars-with-frills in empty space.

These problems of radiation have been discussed in general terms at some length, because such discussions have unfortunately been lacking in the literature of radiation. It seems essential that we should have at least an idea of the conditions that make a problem definite from a mathematical viewpoint, and also that we should recognize the simplifications that are made to reduce practical problems to a form that admits mathematical treatment. 